\title{
Relative Nerve Blocking Properties of Bupivacaine and Ropivacaine in Dogs Undergoing Brachial Plexus Block Using a Nerve Stimulator
}

\author{
Iwao SAKONJU'1)*, Kenichi MAEDA ${ }^{1)}$, Ryoko MAEKAWA ${ }^{2)}$, Rie MAEBASHI ${ }^{3)}$, Tomoko KAKUTA ${ }^{1)}$ and \\ Katsuaki TAKASE ${ }^{1)}$ \\ ${ }^{1)}$ Department of Small Animal Surgery 1, School of Veterinary Medicine, Kitasato University, 23-35-1 Towada, Aomori 034-8628, \\ ${ }^{2)}$ Kawabata Animal Medical Center, 1-11-15 Iwato, Yokosuka, Kanagawa 239-0844 and ${ }^{3)}$ Ohno Dog \& Cat Hospital, 5-2 Kurizaki, \\ Honjo, Saitama 367-0032, Japan
}

(Received 28 January 2009/Accepted 17 May 2009)

\begin{abstract}
In the present study, the efficacy of a nerve stimulator in performing brachial plexus block (BPB) in dogs was investigated. The nerve blocking effects of bupivacaine and ropivacaine for BPB were also compared. Twelve beagles were allocated to groups based on the following treatments: conventional BPB with $0.5 \%$ bupivacaine $(0.5 \%$ BupiM group $)$ or BPB with $0.5 \%$ bupivacaine, $0.5 \%$ ropivacaine or $0.75 \%$ ropivacaine and a nerve stimulator (the $0.5 \%$ BupiS, $0.5 \%$ RopiS and $0.75 \%$ RopiS groups, respectively). After BPB, nerve blocking effects were assessed based on sensory blockade in several cutaneous areas and knuckling. The ratio of full block (blockade in all cutaneous areas) for $0.5 \%$ BupiM was $25 \%$, and that for $0.5 \%$ BupiS was significantly higher, $75 \%$ ( $<<0.05$ ). For the $0.5 \%$ BupiS, $0.5 \%$ RopiS and $0.75 \%$ RopiS groups, the average duration of full block was 387, 184 and 275 min, respectively, and the average duration of knuckling was 703, 460 and $421 \mathrm{~min}$, respectively. The duration of full block and knuckling for the two ropivacaine groups was shorter compared with that of the $0.5 \%$ BupiS group. In conclusion, when using bupivacaine and ropivacaine for BPB in dogs, it is worth noting that there are differences in onset time and duration and that effective perioperative analgesia can be achieved depending on the intended use.

KEY WORDS: brachial plexus block, bupivacaine, canine, nerve stimulator, ropivacaine.
\end{abstract}

J. Vet. Med. Sci. 71(10): 1279-1284, 2009

Brachial plexus block (BPB) is a widely accepted procedure in veterinary medicine to provide surgical analgesia for the front limb distal to the shoulder [14-16, 21]. However, as BPB is often performed blindly using anatomical landmarks, unpredictability of the block and long onset times have been reported [19]. Furthermore, these acataleptic effects preclude the comparison of pharmacological potency between anesthetics. Several reports have described the use of nerve stimulators to locate the brachial plexus, and this approach has significantly improved the attainment of regional blocks in dogs $[8,22]$. Therefore, we assumed that these technical improvements would enable the accurate comparison of pharmacological potency between anesthetics in specific regional anesthesia.

Bupivacaine is a long-acting amide anesthetic and has been used in various types of regional nerve block, infiltration anesthesia and epidural anesthesia to provide long-term perioperative analgesia in small animals [16]. Ropivacaine is a relatively new regional anesthetic with an improved safety profile, particularly with regard to cardiac and central nervous system toxicity, as compared with bupivacaine [9]. In addition, ropivacaine has a lower motor blocking efficacy than bupivacaine $[4,5]$. In humans, it has been reported that bupivacaine and ropivacaine produce similar anesthetic characteristics during BPB $[10,11]$. However, little information is available with regard to the comparative anes-

\footnotetext{
* Correspondence to: SAKonju, I., First Department of Small Animal Surgery, School of Veterinary Medicine, Kitasato University, 23-35-1 Towada, Aomori 034-8628, Japan.

e-mail: sakon@vmas.kitasato-u.ac.jp
}

thetic properties of ropivacaine and bupivacaine in terms of $\mathrm{BPB}$ in dogs.

The aim of this study was to evaluate the nerve blocking effects of bupivacaine and ropivacaine during BPB using a nerve stimulator in normal dogs.

\section{MATERIALS AND METHODS}

Animals: Twelve adult beagles ( 6 males and 6 females, weighing $7.0-10.5 \mathrm{~kg}$ ) that were shown to be clinically healthy on general examination were used. The dogs were used in all groups with a minimum interval of 7 days and were fasted for $12 \mathrm{hr}$ before anesthesia. All protocols in the present study were approved by the Institutional Animal Care and Use Committee of Kitasato University.

Dogs were randomly allocated to the following four groups: the $0.5 \%$ BupiM, 0.5\% BupiS, 0.5\% RopiS and $0.75 \%$ RopiS groups. BPB was performed in the conventional manner using $0.5 \%$ bupivacaine $(0.4 \mathrm{~m} l / \mathrm{kg} ; 0.5 \%$ Marcaine ${ }^{\circledR}$ Injection, AstraZeneca, Osaka, Japan; $n=12$ ) in the $0.5 \%$ BupiM group. BPB was performed using a nerve stimulator and $0.5 \%$ bupivacaine $(0.4 \mathrm{ml} / \mathrm{kg} ; \mathrm{n}=12), 0.5 \%$ ropivacaine $\left(0.4 \mathrm{ml} / \mathrm{kg}\right.$; Anapeine ${ }^{\circledR}$ Injection, AstraZeneca; $\mathrm{n}=12)$ or $0.75 \%$ ropivacaine $(0.4 \mathrm{ml} / \mathrm{kg} ; \mathrm{n}=12)$ in the $0.5 \%$ BupiS, $0.5 \%$ RopiS and $0.75 \%$ RopiS groups, respectively.

Anesthetic procedure for BPB: BPB was performed by continuous rating infusion (CRI) of propofol (Rapinovet ${ }^{\mathbb{R}}$, Schering-Plough, Animal Health, Osaka, Japan) over a short period of time. After bolus propofol administration $(6 \mathrm{mg} /$ $\mathrm{kg}$ ) through the cephalic vein, anesthesia was maintained by 
CRI of propofol $(0.2-0.6 \mathrm{mg} / \mathrm{kg} / \mathrm{min})$ with spontaneous respiration without intubation. After BPB, CRI of propofol was immediately stopped, and the dogs were quickly awakened.

Brachial plexus block: For the 0.5\% BupiM group, BPB was performed according to the method of Quandt et al. [16]. Each dog was positioned in lateral recumbency. While mildly pulling the antebrachium to the caudal side, a needle was placed parallel to the spine from the medial side of the head of the humerus towards the costochondral junction. Once the needle tip was past the head of the humerus, it was confirmed that blood and air did not flow backward through the syringe, and half of $2 \mathrm{mg} / \mathrm{kg} 0.5 \%$ bupivacaine was injected while withdrawing the needle tip to midline of the humerus head. After the first blocking procedure, the needle was removed from the skin, and the same procedure was repeated to inject the remaining half. To ensure that the study conditions were as similar as possible, an insulated electrode needle was used, as in BPB using the nerve stimulator.

In the three groups other than the $0.5 \%$ BupiM group, BPB was performed using a nerve stimulator (TOF Watch ${ }^{\circledR}$ SX, Schering-Plough, Osaka, Japan). Each dog was positioned in the same manner as for conventional BPB, and the anode side of the nerve stimulator was connected to a surface electrode attached to the skin near the upper margin of the stimulation-side scapula; and the cathode side was connected to the insulated electrode needle. The insulated electrode needle was placed in the same manner as in the $0.5 \%$ BupiM group. The stimulation frequency was set at $1 \mathrm{~Hz}$, and the stimulation current was initially set at $5 \mathrm{~mA}$. The needle was advanced until front limb retinacular muscle twitching was induced, and the current was then reduced to $0.5 \mathrm{~mA}$ while positioning the needle in an area with marked twitching; the site of administration was set as the area with residual muscle twitching. If the muscle twitching disappeared when the current was reduced to $0.5 \mathrm{~mA}$, it was determined that the needle tip was not adjacent to a nerve, and the needle was placed again. Just before drug administration, negative pressure was applied to the syringe connected to the stimulation needle in order to ensure that blood and air did not flow backward, and a local anesthetic was then injected. After each procedure, the needle tip was pulled out from the skin, and the same procedure was repeated four times, injecting a quarter of the volume each time. The position of needle placement was not intentionally changed between procedures. To avoid variations in blocking effects due to technical skill, BPB was performed by the same person throughout the study and was completed within 10 min each time. In the present study, the volume of drug administration was $0.4 \mathrm{~m} / / \mathrm{kg}$ in all groups; the drug dose was $3 \mathrm{mg} / \mathrm{kg}$ for the $0.75 \%$ RopiS group and was $2 \mathrm{mg} /$ $\mathrm{kg}$ for the other three groups.

Measurements: The end of BPB was considered to be 0 min. After $\mathrm{BPB}$, the heart and respiration rates were measured at $0,30,60,90,120,180$ and $240 \mathrm{~min}$. The heart rates was determined by auscultation, and the respiration rates was measured by counting thoracic movements.

Skin sensory nerves were assessed based on reaction to nociceptive stimuli in cutaneous areas innervated by each test nerve. Sensory nerves were assessed by the same individual throughout the study. Skin sensory tests were conducted at cutaneous areas innervated by the axillary, musculocutaneous, ulnar, median and radial nerves, and sensory blockade was confirmed by pinching each area using vulsellum forceps.

Sensory tests were conducted at the following cutaneous areas and nerves: 1) the area innervated by the axillary nerve, 2) the area innervated by the musculocutaneous nerve, 3 ) the two areas innervated by the ulnar nerve, 4) the area innervated by the radial nerve and 5) the area innervated by the median nerve. The area innervated by the axillary nerve refers to the lateral aspect of the distal half of the humerus innervated by N. cutaneus brachii lateralis cranialis, a branch of the axillary nerve. The area innervated by the musculocutaneous nerve refers to the medial aspect of the antebrachium, just distal to the elbow, innervated by $\mathrm{N}$. cutaneus antebrachii medialis, a branch of the musculocutaneous nerve. The two areas innervated by the ulner nerve refers to the caudal aspect of the distal half of the antebrachium innervated by N. cutaneus antebrachii caudalis and the dorsolateral aspect of the lateral digit innervated by N. digitalis dorsalis abaxialis $V$, branches of the ulnar nerve. The area innervated by the radial nerve refers to the dorsal aspect of the third digit innervated by Nn. digitales dorsales communes, a branch of the radial nerve. Finally, the area innervated by the median nerve refers to the ventral aspect of the third digit innervated by Nn. digitales palmares communes, a branch of the median nerve.

The above-mentioned tests were conducted at $0,15,30$, 45, 60, 90 and $120 \mathrm{~min}$, and after $120 \mathrm{~min}$, tests were conducted every 60 min until blocking effects disappeared. The examiner used vulsellum forceps to pinch each area, and reactions were graded as follows: -1 point (no reaction at all), 0 points (slight bending of the block-side limb) and 1 point (avoidance reactions such as running away or biting). For each area, the score for the block-side limb was subtracted from the score for the contralateral limb in order to calculate a block score. The onset of blockade was defined as two consecutive tests with block scores of $\geq 1$ point, and the end of blockade was defined as two consecutive tests with block scores of $\leq 0$ points. Full block was defined as a state in which blockade was confirmed by all skin sensory tests. Onset time and duration of full block were then recorded. To assess the quality of sensory nerve block, scores for skin sensory tests were totaled for each measurement period to calculate sensory nerve block scores.

In addition to the sensory test, elicitation of knuckling on the block-side limb was observed, and onset time and duration after BPB were recorded.

Statistical analysis: A $\chi^{2}$-test was used for the full block and knuckling rates. ANOVA and Tukey-Kramer test (post-hoc) were used for heart rate, respiration rate, full block onset time and duration and knuckling onset time and 
duration. Sensory nerve block scores were subjected to a Steel-Dwass multiple comparison test. In all analyses, the level of significance was set at $\mathrm{p}<0.05$.

\section{RESULTS}

Heart and respiration rates: Heart and respiration rates changed almost constantly after the end of BPB in all groups, and no significant differences were seen among the groups in any measurement period (Table 1).

Full block and knuckling rates, and their onset times and durations: The rates of full block and knuckling and their onset times and durations are summarized in Table 2. Because the full block rate for the $0.5 \%$ BupiM group was significantly lower than that for the other groups, the onset time and duration of full block and knuckling were removed from the analysis.

The full block rate for the $0.5 \%$ BupiM group was $25 \%$, but that for the $0.5 \%$ BupiS group was significantly higher, at $75 \%(p<0.05)$. Compared with the $0.5 \%$ BupiM group, the full block rates for the two ropivacaine groups were significantly higher, and full block was achieved in all dogs in the $0.75 \%$ RopiS group. No significant differences were seen among the three groups in which the nerve stimulator was used. In addition, the average onset time of full block was $67 \mathrm{~min}$ for the $0.5 \%$ BupiS group, which was the slowest among the groups. On the other hand, the average onset time for the $0.75 \%$ RopiS group was $35 \mathrm{~min}$, which was significantly faster than that of the $0.5 \%$ BupiS group $(p<0.05)$. The average duration of full block for the $0.5 \%$ BupiS group was the longest at $387 \mathrm{~min}$, and that for the $0.5 \%$ RopiS group was significantly shorter, $185 \mathrm{~min}(\mathrm{p}<0.01)$.

As was the case with full block, knuckling occurred in all dogs in the $0.75 \%$ RopiS group, and there was a significant difference compared with the $0.5 \%$ BupiM group $(\mathrm{p}<0.05)$, but no significant differences were seen compared with the other groups. No significant differences were seen in the onset time of knuckling between the 2 ropivacaine groups, and the onset time of knuckling for the $0.5 \%$ RopiS group was significantly shorter than that for the $0.5 \%$ BupiS group

Table 1. Heart and respiratory rates in dogs undergoing brachial plexus block with $0.5 \%$ bupivacaine or $0.5 \%$ or $0.75 \%$ ropivacaine according to the conventional method or using a nerve stimulator

\begin{tabular}{|c|c|c|c|c|c|c|c|c|c|}
\hline & \multicolumn{9}{|c|}{ Time (min) } \\
\hline & & Pre & 0 & 30 & 60 & 90 & 120 & 180 & 240 \\
\hline \multirow[t]{2}{*}{ 0.5\% BupiM } & HR & $100 \pm 23$ & $111 \pm 29$ & $91 \pm 20$ & $93 \pm 21$ & $91 \pm 20$ & $93 \pm 14$ & $88 \pm 15$ & $89 \pm 14$ \\
\hline & $\mathrm{RR}$ & $28 \pm 9$ & $26 \pm 9$ & $32 \pm 9$ & $29 \pm 8$ & $32 \pm 11$ & $34 \pm 11$ & $27 \pm 6$ & $36 \pm 11$ \\
\hline \multirow[t]{2}{*}{$0.5 \%$ BupiS } & HR & $89 \pm 13$ & $95 \pm 10$ & $97 \pm 11$ & $92 \pm 15$ & $91 \pm 9$ & $90 \pm 20$ & $89 \pm 20$ & $86 \pm 13$ \\
\hline & RR & $33 \pm 9$ & $25 \pm 13$ & $31 \pm 10$ & $32 \pm 7$ & $30 \pm 7$ & $31 \pm 12$ & $32 \pm 11$ & $28 \pm 7$ \\
\hline \multirow[t]{2}{*}{$0.5 \%$ RopiS } & HR & $92 \pm 15$ & $94 \pm 9$ & $99 \pm 17$ & $91 \pm 17$ & $90 \pm 13$ & $85 \pm 9$ & $81 \pm 21$ & $79 \pm 10$ \\
\hline & $\mathrm{RR}$ & $33 \pm 13$ & $21 \pm 4$ & $26 \pm 8$ & $26 \pm 12$ & $31 \pm 11$ & $35 \pm 13$ & $30 \pm 9$ & $31 \pm 7$ \\
\hline \multirow[t]{2}{*}{$0.75 \%$ RopiS } & HR & $107 \pm 24$ & $100 \pm 14$ & $98 \pm 19$ & $93 \pm 16$ & $99 \pm 15$ & $102 \pm 27$ & $105 \pm 24$ & $92 \pm 22$ \\
\hline & $\mathrm{RR}$ & $32 \pm 11$ & $24 \pm 10$ & $30 \pm 9$ & $28 \pm 6$ & $31 \pm 6$ & $32 \pm 7$ & $30 \pm 8$ & $29 \pm 6$ \\
\hline
\end{tabular}

Data are means $\pm \mathrm{SD}$.

$\mathrm{HR}$, heart rate; $\mathrm{RR}$, respiratory rate.

$0.5 \%$ BupiM: brachial plexus block was performed in a conventional manner using $0.5 \%$ bupivacaine. $0.5 \%$ BupiS, $0.5 \%$ RopiS and $0.75 \%$ RopiS: brachial plexus block was performed using a nerve stimulator and $0.5 \%$ bupivacaine, $0.5 \%$ and $0.75 \%$ ropivacaine, respectively.

Table 2. Results of brachial plexus block with $0.5 \%$ bupivacaine, or $0.5 \%$ or $0.75 \%$ ropivacaine, according to the conventional method or using a nerve stimulator

\begin{tabular}{lcccc}
\hline & \multicolumn{3}{c}{ Groups } \\
& $\begin{array}{c}0.5 \% \text { BupiM } \\
(\mathrm{n}=12)\end{array}$ & $\begin{array}{c}0.5 \% \text { BupiS } \\
(\mathrm{n}=12)\end{array}$ & $\begin{array}{c}0.5 \% \text { RopiS } \\
(\mathrm{n}=12)\end{array}$ & $\begin{array}{c}0.75 \% \text { RopiS } \\
(\mathrm{n}=12)\end{array}$ \\
\hline No. of dogs exhibiting full block & $3(25 \%)$ & $9(75 \%)^{*}$ & $11(92 \%)^{* *}$ & $12(100 \%)^{* * *}$ \\
Onset time of full block (min) & $100 \pm 17^{\#}$ & $67 \pm 34$ & $50 \pm 24$ & $35 \pm 10^{\dagger}$ \\
Duration of full block (min) & $340 \pm 46^{\#}$ & $387 \pm 153$ & $184 \pm 103^{\dagger \dagger}$ & $275 \pm 115$ \\
No. of dogs exhibiting knuckling & $7(58 \%)$ & $10(83 \%)$ & $11(92 \%)$ & $12(100 \%)^{*}$ \\
Onset time of knuckling (min) & $77 \pm 93^{\#}$ & $33 \pm 25$ & $16 \pm 4^{\dagger}$ & $19 \pm 7$ \\
Duration of knuckling (min) & $480 \pm 112^{\#}$ & $703 \pm 91$ & $460 \pm 86^{\dagger \dagger}$ & $421 \pm 122^{\dagger \dagger}$ \\
\hline
\end{tabular}

Data are means \pm SD.

$*, * *, * * *$ : significant differences vs. 0.5\% BupiM (*,** and ***: $\mathrm{P}<0.05, \mathrm{P}<0.01$ and $\mathrm{P}<0.001$, respectively). ${ }^{\dagger},{ }^{\dagger},{ }^{\dagger \dagger}$ : significant differences vs. $0.5 \%$ BupiS $\left({ }^{\dagger},{ }^{\dagger}\right.$ and ${ }^{\dagger \dagger}: \mathrm{P}<0.05, \mathrm{P}<0.01$ and $\mathrm{P}<0.001$, respectively). $0.5 \%$ BupiM: brachial plexus block was performed in a conventional manner using $0.5 \%$ bupivacaine. $0.5 \%$ BupiS, $0.5 \%$ RopiS and $0.75 \%$ RopiS: brachial plexus block was performed using a nerve stimulator and $0.5 \%$ bupivacaine, $0.5 \%$ and $0.75 \%$ ropivacaine, respectively. ${ }^{\#}$ : Onset time and duration of full block and knuckling in $0.5 \%$ BupiM were excluded from the statistical analysis. 


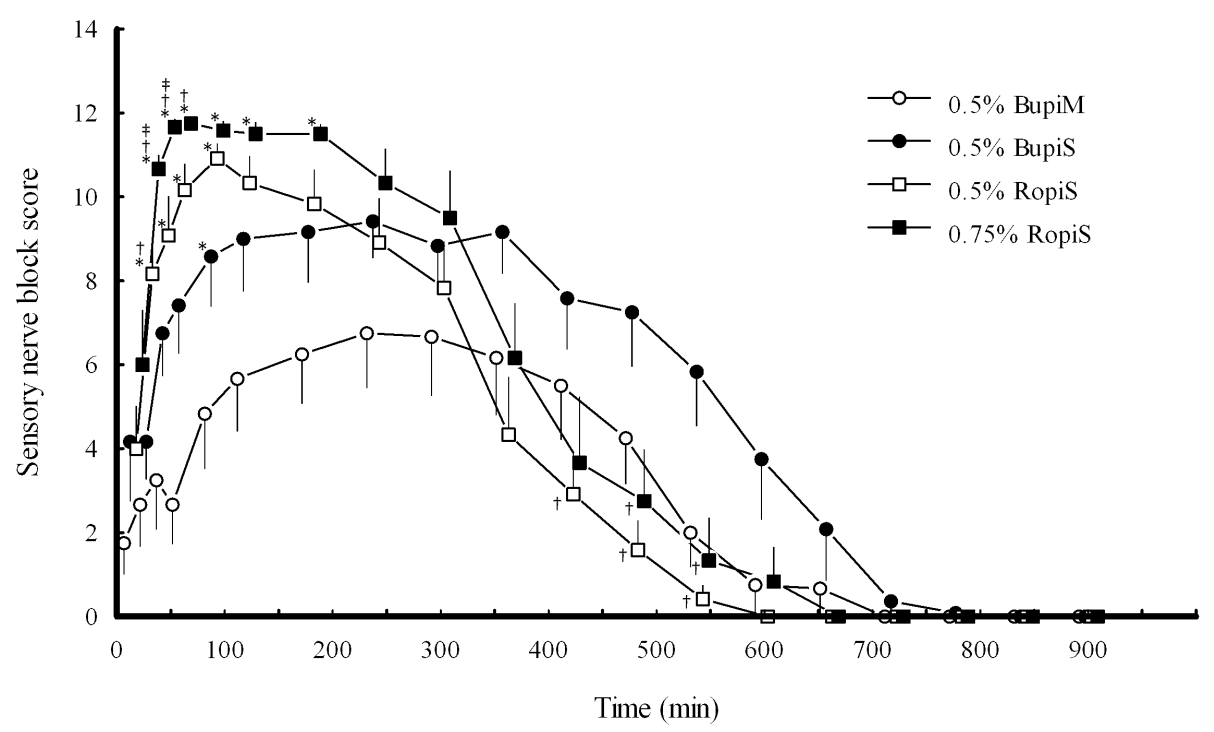

Fig. 1. Changes in sensory nerve block score. Data are means \pm SD. The symbols, $* \dagger$ and $\ddagger$ indicate significant differences between the $0.5 \%$ BupiM, $0.5 \%$ BupiS and $0.5 \%$ RupiS groups, respectively. $0.5 \%$ BupiM: brachial plexus block was performed in a conventional manner using $0.5 \%$ bupivacaine $0.5 \%$ BupiS, $0.5 \%$ RopiS and $0.75 \%$ RopiS: brachial plexus block was performed using a nerve stimulator and $0.5 \%$ bupivacaine, $0.5 \%$ and $0.75 \%$ ropivacaine, respectively.

$(\mathrm{p}<0.05)$. In addition, the average duration of knuckling for the $0.5 \%$ BupiS group was the longest, $703 \mathrm{~min}$, and this was significantly longer than those in the $0.5 \%$ RopiS and $0.75 \%$ RopiS groups $(\mathrm{p}<0.001)$.

Changes in sensory nerve block scores: Figure 1 shows the changes in sensory nerve block scores in each measurement period. During the observation period, the average score for the $0.5 \%$ BupiS group was consistently higher compared with the $0.5 \%$ BupiM group, but significant differences were only seen at $90 \mathrm{~min}(\mathrm{p}<0.05)$. The average score for the 2 ropivacaine groups was higher until $180 \mathrm{~min}$ compared with the $0.5 \%$ BupiS group, but rapidly decreased thereafter, and after $360 \mathrm{~min}$, the average score for the $0.5 \%$ BupiS group was higher. Throughout the observation period, the average scores for the $0.5 \%$ and $0.75 \%$ RopiS groups changed in a comparable manner, and a significant difference was only seen at 30 and $45 \mathrm{~min}(\mathrm{p}<0.05)$.

\section{DISCUSSION}

There have been several reports on BPB using a nerve stimulator in small animals $[8,22,23]$. However, as these studies did not compare blocking effects with or without a nerve stimulator, the usefulness of nerve stimulators has not been demonstrated. With conventional BPB, blocking effects are unstable, and it has thus been difficult to compare analgesic effects among drugs. When using a nerve stimulator, an electric current discharged from the needle tip induces peripheral nerve depolarization. If the needle tip is away from the nerve, lowering the stimulation current eliminates muscle twitching. On the other hand, if the needle tip is close to the nerve, muscle twitching still occurs with a weak current, thus making it possible to inject a drug solution near the nerve.

Ford et al. [7] investigated the distance between the insulated needle and the nerve and reported that the average distance to the feline saphenous nerve required to achieve complex activity potentials with an average of $0.5 \mathrm{~mA}$ was $0 \mathrm{~cm}$. Eifert et al. [2] also performed BPB using a nerve stimulator in humans and achieved a success rate of $85 \%$, but the minimum stimulation current was $0.4 \mathrm{~mA}$ in successful cases and $0.6 \mathrm{~mA}$ in failed cases, thus suggesting that it is necessary to lower the current to $0.5 \mathrm{~mA}$ in order to determine the location of peripheral nerves. Based on these reports, the final stimulation current in this study was set at $0.5 \mathrm{~mA}$.

In the present study, the full block rate (an indicator for sensory blocking effect) for the $0.5 \%$ BupiM group was $25 \%$, but that for the $0.5 \%$ BupiS group was significantly higher, $75 \%(\mathrm{p}<0.05)$. In addition, the sensory nerve block score for the $0.5 \%$ BupiS group was higher compared with the $0.5 \%$ BupiM group throughout the study. This shows that use of a nerve stimulator markedly improves the accuracy of BPB. Therefore, in the present study, it was possible to more accurately compare efficacy between bupivacaine and ropivacaine.

With regard to the blocking effects of bupivacaine and ropivacaine on peripheral nerves, most studies have reported that the onset time and duration of blockade are comparable at similar doses [3, 12, 13, 17]. However, Åkerman et al. [1] administered comparable doses of bupivacaine or ropivacaine epidurally to guinea pigs and reported 
that the blockage duration for ropivacaine was clearly shorter compared with that of bupivacaine. They indicated that this is because the low lipid affinity of ropivacaine quickens its dissociation from neural tissue, thus shortening the effect duration. Conversely, the low lipid affinity of ropivacaine to the external neural lipid quickens its transition to nerve action sites, thus resulting in rapid blocking effects [18]. The results of the present study were in agreement with these reports.

In the present study, comparison of the $0.5 \%$ BupiS and $0.5 \%$ RopiS groups revealed no significant differences in full block onset time, but the blockade duration for the $0.5 \%$ RopiS group was significantly shorter. In the $0.5 \%$ BupiS group, full block was achieved in an average of $67 \mathrm{~min}$, but in the $0.75 \%$ RopiS group, full block was achieved in only $35 \mathrm{~min}$. Therefore, it is necessary to consider the possibility that sufficient analgesic effects are not achieved by commencement of surgical intervention in BPB using bupivacaine, even if a nerve stimulator is used. On the other hand, the full block duration for the $0.5 \%$ BupiS group was the longest among the four groups, which suggests that bupivacaine is more effective with regard to postoperative analgesia.

In the $0.75 \%$ RopiS group, full block was achieved in all dogs, and shorter onset time and longer duration were observed compared with the $0.5 \%$ RopiS group. The dosage of ropivacaine in the $0.75 \%$ RopiS group was $3 \mathrm{mg} / \mathrm{kg}$ in the present study. Feldman et al. [6] reported that the intravenous dose required to induce twitching is $4.9 \mathrm{mg} / \mathrm{kg}$ for ropivacaine and $4.3 \mathrm{mg} / \mathrm{kg}$ for bupivacaine. They also observed increases in respiratory and heart rates with central nervous system toxicity after intravenous administration of bupivacaine and ropivacaine in conscious dogs. In the present study, there were no significant changes in heart and respiratory rates, and adverse effects indicating central nervous system toxicity, such as excitement, tremor and convulsion, were not observed in any groups. In addition, Tucker et al. [20] reported that the order of absorption speeds for identical local anesthetics is intercostal $>$ sacroepidural $>$ lumber epidural $>$ brachial plexus $>$ subarachnoid $>$ infiltration anesthesia $>$ subcutaneous. Considering that the absorption speed of local anesthetics administrated to the brachial plexus is slow, $0.75 \%$ ropivacaine at $0.4 \mathrm{ml} /$ $\mathrm{kg}$ with BPB is sufficiently safe, at least in dogs with the body weight range used in this study.

Although we assumed that the indices for full block exclusively reflected sensory nerve blocking effects, knuckling involves both motor nerves and sensory nerves. The elicitation of knuckling is mainly influenced by radial nerve blocking. Radial nerves are mixed nerves containing both sensory and motor nerves, and thus the exclusive blocking effects of the anesthetics on motor nerves were not clearly observed in this study. However, dogs in each groups showed knuckling before full block, and the mean duration of knuckling was distinctly longer than that of full block. These results indicate that motor function is more suscepti- ble to BPB than nociceptive perception. Furthermore, the average duration of full block for the $0.75 \%$ RopiS group was longer than that for the $0.5 \%$ RopiS group, while the average duration of knuckling was shorter. Thus, the duration of knuckling is not necessarily proportional to the duration of full block, suggesting that both the anesthetic and concentration provide specific blocking effects on motor and sensory nerves with regard to strength and duration.

Compared with bupivacaine, ropivacaine is reported to be sensory nerve selective and to possess weak motor blocking effects because of its weaker effects on Ab fibers [13]. Feldman et al. [4] compared the effects of ropivacaine and bupivacaine by injection into the sciatic nerve region in rats and the lumbar epidural or subarachnoid space in dogs and assessment based on the extent of motor nerve block by akinesia in hindlimbs. The results showed that, at comparable doses, the length of time required to paralyze motor nerves and the effect duration were shorter for ropivacaine. With regard to the motor function, our results were consistent with theirs. However, Feldman et al. did not investigate sensory blocking effects, and thus, relative sensory nerve selectivity has not been clarified. In the present study, onset time and duration of knuckling for the 0.5\% RopiS group were significantly shorter compared with the $0.5 \%$ BupiS group $(\mathrm{p}<0.001)$, with the onset time and duration for the $0.5 \%$ BupiS group being $48 \%$ and $65 \%$, respectively. However, the average duration of full block in the $0.5 \%$ RopiS group was also $52 \%$ shorter compared with that of the $0.5 \%$ BupiS group. Therefore, the relative sensory nerve selectivity of ropivacaine for motor function was not confirmed in this study.

The canine brachial plexus consists of at least 5 nerves. Therefore, plexus blockade was assessed by skin sensory loss at six cutaneous areas innervated by the five nerves. In the present study, two cutaneous areas were used for sensory tests in the ulnar nerve alone because we found that it was most difficult to obtain sensory block in areas innervated by the ulnar nerve in preliminary experiments. Elicitation and duration of full block tended to be limited with ulnar nerve block in the $0.5 \%$ BupiM group. This may be because the anatomical location of the ulnar nerve in the brachial plexus is relatively far from the needling point. However, the nerve stimulator improved the quality of BPB, and full block was achieved in all dogs that were administered $0.75 \%$ ropivacaine using the nerve stimulator. We also demonstrated that ropivacaine produced stronger but shorter blocking effects than bupivacaine and that bupivacaine produced longer lasting effects following delayed onset of blocking effects. These results clarify that a nerve stimulator is very useful for BPB in dogs. At the same time, the characteristic blocking effects of ropivacaine and bupivacaine in BPB were clarified. When performing BPB for perioperative analgesia, a more effective block can be achieved by taking into account the pharmacological differences between the two local anesthetics. 


\section{REFERENCES}

1. Åkerman, B., Hellberg. I.B. and Trossvik, C. 1988. Primary evaluation of local anaesthetic properties of the amino amide agent ropivacaine (LEA 103). Acta. Anaesthesiol. Scand. 32: 571-578.

2. Eifert, B., Hähenel, J. and Kustermann, J. 1994. Axillary block. A prospective study of the success of blockade using a nerve stimulator. Anaethesist 43: 780-785.

3. Fanelli, G., Casati, A., Beccaria, P., Aldegheri, G., Berti, M., Tarantino, F. and Torri, G. 1998. A double-blind comparison of ropivacaine, bupivacaine, and mepivacaine during sciatic and femoral nerve blockade. Anesth. Analg. 87: 597-600.

4. Feldman, H.S. and Covino, B.G. 1988. Comparetive motorblocking effects of bupivacaine and ropivacaine, a new amino amido local anethetic, in the rat and dog. Anesth. Analg. 67: 1047-1052.

5. Feldman, H.S. Dvoskin, S., Arthur G.R. and Doucette, A.M. 1996. Antinociceptive and motor-blocking efficacy of ropivacaine and bupivacaine after epidural administration in the dog. Reg. Anesth. 21: 318-326.

6. Feldman, H.S., Richard, G., Pitkanen, M., Hurley, R., Doucette, A.M. and Covino, B.G. 1991. Treatment of acute systemic toxicity after the rapid intravenous injection of ropivacaine and bupivacaine in the conscious dog. Anesth. Analg. 73: 373-384.

7. Ford. D. J., Pither. C. and Raj, P. 1984. Comparison of insulated and uninsulated needles for locating peripheral nerve with a peripheral nerve stimulator. Anesth. Analg. 63: 925-928.

8. Futema, F., Fantoni, T. D., Auler, O. C. J. Jr., Cortppassi, R. G. S., Acaui, A. and Stopiglia. 2002. A new brachial plexus block technique in dogs. Vet. Anesth. Analg. 29: 133-139.

9. Hansen, T.G. 2004. Ropivacaine:a pharmacological review. Expert Rev. Neurotherapeutics. 4: 781-791.

10. Hickey, R., Hoffman, J. and Ramamurthy, S. 1991. A comparison of ropivacaine $0.5 \%$ and bupivacaine $0.5 \%$ for brachial plexus block. Anesthesiology 74: 639-642.

11. Hickey, R., Rowley, C.L., Candido, K.D., Hoffman, J., Ramamurthy, S. and Winnie, A.P. 1992. A comparative study of $0.25 \%$ ropivacaine and $0.25 \%$ bupivacaine for brachial plexus block. Anesth. Analg. 75: 602-606.
12. Klein, S.M., Greengrass, R.A., Steele. S.M., D’Ercole, F.J., Speer, K.P., Gleason, D.H., DeLong, E.R. and Warner, D.S. 1998. A comparison of $0.5 \%$ bupivacaine, $0.5 \%$ ropivacaine, and $0.75 \%$ ropivacaine for interscalene brachial plexus block. Anesth. Analg. 87: 1316-1319.

13. Mcglade, D.P., Kalpokas, M.V., Moony, P.H., Chamley, D., Mark, A.H. and Torda, T.A. 1998. Acomparison of $0.5 \%$ ropivacaine and $0.5 \%$ bupivacaine for axillary brachial plexus anesthesia. Anesth. Intensive Care. 26: 515-520.

14. Moens, N.M.M. and Caulkett, N.A. 2000. The use of a catheter to provide brachial plexus block in dogs. Can. Vet. J. 41: 685689.

15. Nutt, P. 1962. Brachial plexus analgesia in the dog. Vet. Rec. 74: 874-877.

16. Quandt, J.E. and Rawlings, C.R. 1996. Reducing postoperative pain for dogs:Local anesthetic and analgesic techniques. Comp. Contin. Educ. Pract. Vet. 18: 101-111.

17. Ræder, J.C., Dresdahl, S., Klaastad, $\theta$. , Kvalsvik, O., Isaksan, B., Storemskag, E., Mowinckel, P., Berghem, R. and Selander, D. 1999. Axillary brachial plexus block with ropivacaine 7.5 $\mathrm{mg} / \mathrm{ml}$. Acta. Anaesthesiol. Scand. 43: 794-798.

18. Sia, S., Bartoli, M., Lepri, A., Marchini, O. and Ponsecchi, P. 2000. Multiple-Injection Axillary brachial plexus block: A comparison of two methods of nerve location- nerve stimulation versus paresthesia. Anesth. Analg. 91: 647-651.

19. Skarda, R.T. 1996. Local and regional anesthetic and analgesic techniques: Dogs. pp. 426-447. In: Lumb \& Jones' Veterinary Anesthesia. 3rd ed. (Thurmon, J.C., Tranquilli, W.J. and Benson, G.J. eds.), Williams \& Wilkins, Baltimore.

20. Tucker, G. T. and Mather, L. E. 1979. Clinical pharmacokinetics of local anesthetics. Clin. Pharmacokinet. 4: 241-278.

21. Tufvesson, G. 1951. Anesthesi av plexus brachialis. Nord. Vet. Med. 3: 183-193.

22. Wenger, S. 2004. Brachial plexus block using electrolocation for pancarpal arthrodesis in a dog. Vet. Anesth. Analg. 31: 272275.

23. Wenger, S., Moens, Y., Jäggin, N. and Schatzmann, U. 2005. Evaluation of the analgesic effect lidocaine and bupivacaine used to provide a brachial plexus block for front limb surgery in 10 dogs. Vet. Rec. 156: 639-642. 